\title{
The Impact of COVID-19 Pandemic on the Interventional Cardiology Workload in Romania in 2020
}

\author{
Cristian UDROIU ${ }^{a}$, Lucian PREDESCU ${ }^{\text {b }}$
}

aEmergency University Hospital, Bucharest, Romania

b"Prof. Dr. C. C. Iliescu” Emergency Institute for Cardiovascular Diseases, Bucharest, Romania

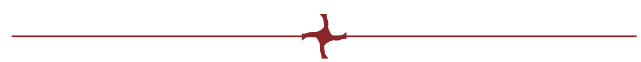

\begin{abstract}
Coronavirus disease has overwhelmed the healthcare systems around the globe at an unprecedented level. The same was observed in the Romanian medical system. Interventional cardiology units have experienced a dramatic reduction in elective and urgent interventional procedures. The present report highlights the total number of coronary interventions, peripheral interventions, and interventions for structural heart diseases that were performed in 2020 in Romania in comparison to 2019.
\end{abstract}

Keywords: interventional cardiology, percutaneous coronary interventions, stents, COVID-19.

\section{INTRODUCTION}

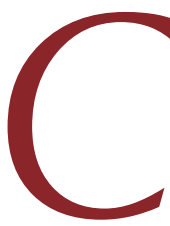

Toronavirus disease, commonly known as COVID-19, has overwhelmed healthcare systems around the globe at an unprecedented level. The same was observed in the Romanian medical system. Interventional cardiology units have experienced a dramatic reduction in elective and urgent interventional procedures. The magnitude of this reduction is difficult to estimate, being a situation never encountered by most doctors of the younger generations. This article summarizes the data about the number of different interventional procedures performed during 2020 in Romania by comparison with data already published until 2019 (1-6).

Like in 2019, all members from interventional cardiology centers in Romania were requested to fill a form about the total number of various procedures performed during 2020. The request was conducted by the president of Interventional Cardiology Working Group of the Romanian Society of Cardiology. 36 of 38 public and private interventional cardiology centers in Romania responded to our request and provided the necessary data.

Table 1 summarizes the general data from 2020 and 2019 and the percentage difference between these two years. In 2020, the number

Address for correspondence:

Dr. Cristian A. Udroiu, President of Interventional Cardiology Working Group of the Romanian Society of Cardiology

Emergency University Hospital, Bucharest Romania

Email: cristiudroiu@yahoo.com

Article received on the $7^{\text {th }}$ of December 2021 and accepted for publication on the $16^{\text {th }}$ of December 2021 


\begin{tabular}{|l|l|l|l|l|}
\hline Year & $\mathbf{2 0 2 0}$ & $\mathbf{2 0 1 9}$ & $\uparrow$ or $\downarrow$ & Percentage (\%) \\
\hline Hospital & & & & \\
\hline Number of cathlabs & 52 & 53 & & \\
\hline Number of independent operators & 132 & 132 & & \\
\hline Number of in training operators & 51 & 58 & & \\
\hline Coronary interventions & & & & \\
\hline & & & & \\
\hline Coronary angiogram & 29824 & 43164 & $\downarrow$ & 44.73 \\
\hline Total number of PCI & 15879 & 21783 & $\downarrow$ & 37.18 \\
\hline PCI without stents & 1116 & 1306 & $\downarrow$ & 17.03 \\
\hline PCI with stents & 14608 & 20015 & $\downarrow$ & 37.01 \\
\hline STEMI with primary PCI & 6587 & 8281 & $\downarrow$ & 25.72 \\
\hline PCI left main & 553 & 961 & $\downarrow$ & 73.78 \\
\hline CTO & 350 & $\mathrm{UR}$ & $\mathrm{UR}$ & $\mathrm{UR}$ \\
\hline Stents per patient ${ }^{2}$ & 1.454 & 1.469706 & $\downarrow$ & 1.08 \\
\hline Total number of stents & 21975 & 32055 & $\downarrow$ & 45.87 \\
\hline Total number of DES & 21550 & 30207 & $\downarrow$ & 40.17 \\
\hline DES/stents ratio (\%) & 98 & 94 & $\uparrow$ & 4.08 \\
\hline Cutting, scoring & 182 & 196 & $\downarrow$ & 7.69 \\
\hline Rotational atherectomy & 79 & 58 & $\uparrow$ & 26.58 \\
\hline Shockwave & 15 & $\mathrm{UR}$ & $\mathrm{UR}$ & $\mathrm{UR}$ \\
\hline FFR/IFR & 238 & 360 & $\downarrow$ & 51.26 \\
\hline IVUS & 75 & 145 & $\downarrow$ & 93.33 \\
\hline OCT & 72 & 148 & $\downarrow$ & 105.56 \\
\hline & & & & \\
\hline Peripheral interventions & & & & \\
\hline Peripheral PTA & 3114 & 1796 & $\downarrow$ & 61.22 \\
\hline Renal PTA & 59 & 93 & $\downarrow$ & 57.63 \\
\hline Carotid PTA & 213 & 382 & $\downarrow$ & 79.34 \\
\hline Total number of peripheral stents & 841 & 1404 & $\downarrow$ & 66.94 \\
\hline Peripheral angiography & 5184 & 6515 & $\downarrow$ & 25.68 \\
\hline & & & & \\
\hline Other interventions & & & & \\
\hline TAVI & 494 & 428 & $\uparrow$ & 13.36 \\
\hline MitraClip & 3 & 3 & $=$ & 0 \\
\hline Aortic endoprothesis & 91 & 113 & $\downarrow$ & 24.18 \\
\hline Interventions in structural heart disease & 171 & 140 & $\uparrow$ & 18.13 \\
\hline & & & \\
\hline & & & \\
\hline
\end{tabular}

${ }^{1}$ Total number of elective and urgent PCIs

${ }^{2}$ It will represent the ratio between the total number of implanted stents and the total number of patients undergoing PCI

${ }^{3}$ Number of peripheral angioplasty (lower and upper limbs artery, not including carotid arteries)

${ }^{4}$ Interventions in cardiac structural diseases: percutaneous closures of DSA, DSV, PFO, arterial duct, balloon valvulotomies, left atrial appendage closure

( $\mathrm{PCI}=$ percutaneous coronary intervention, STEMI=ST segment elevation myocardial

TABLE 1

Reported data of different

infarction, DES=drug eluting stent, BVS=bioresorbable vascular scaffold, FFR=fractional interventional flow reserve, IFR=instantaneous wave-free ratio, IVUS=intravascular ultrasound, procedures by all OCT=optical coherence tomography, PTA=percutaneous transluminal angioplasty, Interventional TAVI=transcatheter aortic valve implantation, $\mathrm{CTO}=$ chronic total occlusion, $\uparrow$ - increase, Cardiology Centers in $\downarrow$ - decrease) 

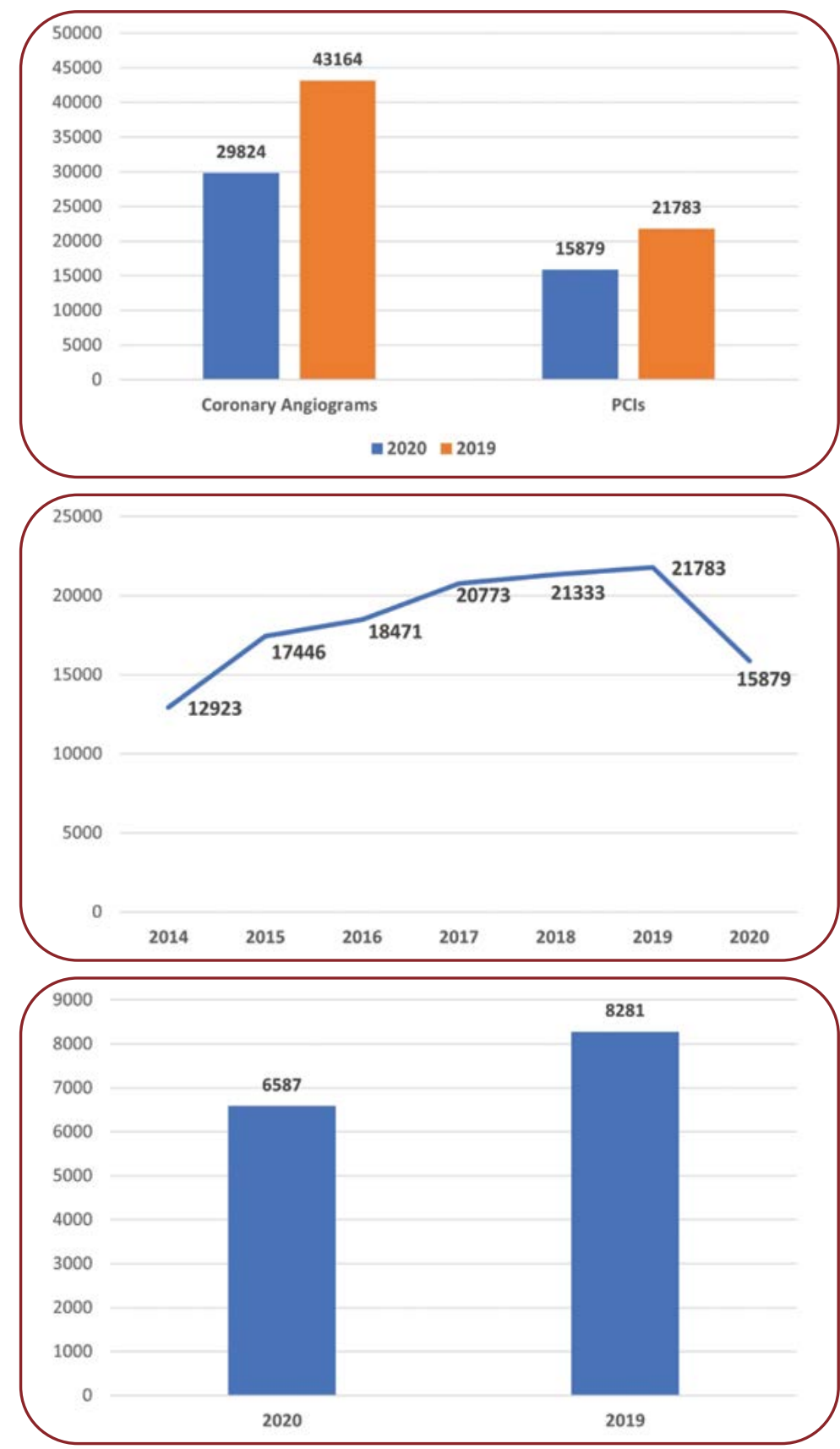

FIGURE 1. Total number of coronary angiograms and percutaneous coronary interventions (PCIs) performed in 2019 and 2020 in Romania
FIGURE 2. Total number of percutaneous coronary interventions from 2014 to 2020 of coronary angiograms decreased by $44.7 \%$, from 43.164 in 2019 to 29.824 in 2020 . The total number of percutaneous coronary interventions (PCls) was reduced by $37.18 \%$ from 21783 in 2019 to 15879 in 2020. This data includes the elective and urgent PCls (Figure 1). In Figure 2 we can see the upward trend of the yearly number of PCls from 2014 to 2019. The number of PCls performed in 2020 fell towards the 2014-2015 levels.

Similarly to the decrease of elective procedures, the total number of primary PCls in STEMI patients also decreased by $25 \%$ in 2020 , from 8281 in 2019 to 6587 in 2020 (Figure 3). Although the magnitude of the decrease in the total number of primary PCls was lower when compared to elective PCls, it still represented an important decrease that raises important problems related to the treatment of this subgroup of patients.

Although complex coronary procedures, like left main $\mathrm{PCl}$, seriously decreased by almost $74 \%$ in 2020 compared to 2019, the number of implanted stents per patient remained almost un- 


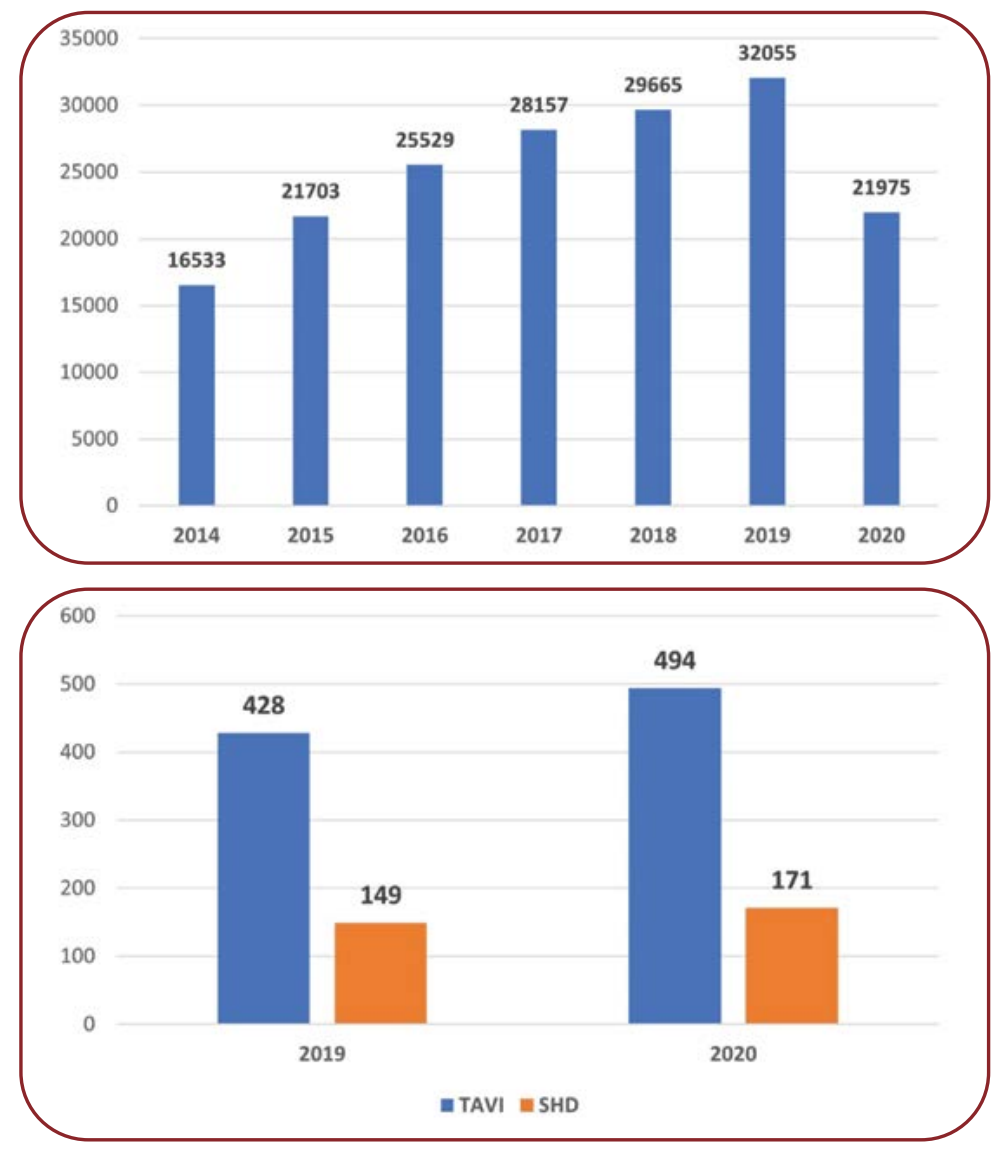

FIGURE 4. Total number of implanted stents from 2014 to 2020
FIGURE 5. Total number of TAVI and SHD interventions in 2019 and 2020 (TAVI=transcatheter aortic valve implantation, SHDs=structural heart diseases) changed. However, the absolute number of implanted stents decreased by $45.8 \%$ in 2020 compared to 2019. In Figure 4 we can see that the number of stents implanted in 2020 is similar to the one reported in 2015.

Decreases between 51\% and 100\% were registered in 2020 in terms of number of intracoronary physiology and imaging investigations as well as peripheral angioplasties, compared to 2019 (Table 1).

The only field that still developed in 2020 was that of structural heart diseases and TAVI interventions. The number of transcatheter aortic valve implantation (TAVI) procedures doubled every year between 2015-2019 and the trend was maintained in 2020, with an increase by 13.3\% compared to 2019 (Figure 5) (1-6). The number of procedures performed in structural heart diseases (SHD) increased by $18.1 \%$ between 2019 of 2020 (Figure 5).

\section{CONCLUSION}

020 was a difficult year for medical activity due to the COVID-19 pandemic. The inter- ventional cardiology activity in Romania was considerably impacted. Although the number of most types of interventional cardiology procedures significantly decreased in 2020 compared to 2019 , some progress was observed in terms of TAVI and SHD interventions.

There are a few possible explanations for this situation. First of all, hospitals reduced the elective procedure due the lack of available beds. The medical was mainly engaged in managing COVID-19 patients, whilst deferring for "chronic" patients. Resources were dedicated to intensive care unit, for protection equipment and for the expensive treatment of COVID-19 patients. Also, patients were reluctant to address hospitals for their cardiac problems medical checks.

On the other hand, the rise in the number of structural heart disease procedures by postponing their regular can be explained by the growing number of interventional centers and increasing experience of the medical teams.

Conflicts of interest: none declared.

Financial support: none declared. 


\section{R $_{\text {EFeRENCes }}$}

1. Predescu L, Postu M. The workload of the Interventional Cardiology Centers from Romania: a 2019 overview and 2014-2019 trends.

Romanian Journal of Cardiology 2020;30:423-432.

2. Predescu L, Postu M. The workload of the Interventional Cardiology Centers from Romania: a 2018 overview and 2014-2018 trends.

Romanian Journal of Cardiology
2019;29:415-421.

3. Predescu L, Postu M. The activity of the Interventional Cardiology Centers in Romania: a 2017 overview and 2014-2017 trends.

Romanian Journal of Cardiology 2018;28:147-153.

4. Postu M, Predescu L, Mot S. The activity of the interventional cardiology centers in Romania: a 2016 overview and 2014-2016 trends.

Romanian Journal of Cardiology
2017;27:359-368.

5. Postu M, Predescu L, Mot S. The activity of the Interventional Cardiology Centers in Romania: a 2015 overview.

Romanian Journal of Cardiology 2016;26:298-309.

6. Postu M, Uşurelu C, Predescu L, et al. Romanian interventional cardiology centers, 2014-2015: an overview. Romanian Journal of Cardiology 2015;25:170-174. 